\title{
Fast and slow domino regimes in transient network dynamics
}

\author{
Peter Ashwin, ${ }^{1,2}$ Jennifer Creaser, ${ }^{1,2}$ and Krasimira Tsaneva-Atanasova ${ }^{1,2,3}$ \\ ${ }^{1}$ Department of Mathematics, University of Exeter, Exeter EX4 4QF, United Kingdom \\ ${ }^{2}$ EPSRC Centre for Predictive Modelling in Healthcare, University of Exeter, Exeter EX4 4QJ, United Kingdom \\ ${ }^{3}$ Living Systems Institute, University of Exeter, Exeter EX4 4QD, United Kingdom
}

(Received 24 January 2017; published xxxxxx)

58 a variety of applications. This includes: (a) signal propagation by sequential switching between asymmetric stable states 59 (observed experimentally in chains of bistable electronic 60 circuits [6] or in cases where the bistability is noise induced 61 [7]), (b) waves along unidirectionally coupled chains (or 62 lattices) of bistable nodes with forcing at one end [8], (c) 63 photoinduced phase transitions in spin-crossover materials 64 with bistable dynamic potentials [9-11], (d) avalanches of gene 65 activation in gene regulatory pathways to drive cell differentia- 66 tion/development/cancer [12,13], or (e) cell fate in biofilm for- 67 mation [14]. Other applications that could benefit from a better 68 understanding of similar transient dynamics induced by noise 69 include (a) the contagion of bank defaults in a system of finan- 70 cial institutions interconnected by mutual loans [15-18], (b) 71 interconnections between "tipping elements" [19-21], (c) the 72 role of spreading of abnormal large-amplitude oscillators in the 73 modeling onset of epileptic seizures [22,23], (d) multiple organ 74 failure [24], or (e) cascading failures in power systems [25]. $\quad 75$

The role of coupling strength in noise-induced transitions 76 on networks is considered by Refs. [26,27] for idealized 77 symmetric bistable systems. Neiman [28] shows similar 78 synchronization effects in coupled stochastic bistable systems 79 and Ref. [29] shows them in coupled ratchet systems. The 80 authors of Refs. [26,27] give rigorous mathematical results that 81 identify the existence of different regimes of synchronization 82 of escapes in the low noise limit that can be linked to changes 83 in the structure of the underlying system attractors (see, for ex- 84 ample, Ref. [30] for some review of the role of coupling in the 85 noise-free context). In particular, Ref. [26] identifies the most 86 likely sequences of escape and how their probabilities change 87 qualitatively with coupling strength: There can be synchro- 88 nized transitions in the strong coupling limit. Many properties 89 of the transitions can be understood using Friedlin-Wentzell 90 methodology and the Eyring-Kramers formula [31-33] to 91 study the pathwise properties of transitions between attractors. 92

We show in the context of asymmetric potentials that ${ }_{93}$ there are typically several qualitatively different regimes 94 in the transient sequences of escapes. These regimes of 95 
96 weak, intermediate, and strong coupling, and the intermediate case may be quite complicated, but in general there are qualitative changes in behavior for the weak noise limit that can be characterized in terms of bifurcations of steady states of the noise-free system. As a row of toppling dominos depends on the properties and spacing of the dominos [34], we identify different domino effects that can be characterized by different 103 coupling regimes. Specifically, we identify "slow domino" and "fast domino" regimes corresponding to intermediate and strong coupling regimes, respectively. Within these different regimes, certain sequences of escape may be preferred by the coupling, and the distribution of times to the next escape may have significant deviations from the exponential.

120 and the distribution of timings within the sequence of escapes 121 can be greatly affected.

$$
\dot{x}=f(x, v):=-(x-1)\left(x^{2}-v\right),
$$

124 so that $f=-V^{\prime}(x)$ with potential $V(x)=\frac{1}{4} x^{4}-\frac{1}{3} x^{3}+$ $125 v\left(x-\frac{1}{2} x^{2}\right)$. We suppose that the nodes are coupled into a 126 network and subjected to additive noise. For $0<v \ll 1$ the 127 stable states are not interchangeable by any symmetry. There is 128 a quiescent attractor at $x=x_{Q}:=-\sqrt{v}$ and an active attractor 129 at $x=x_{A}:=1$; there is an unstable separating equilibrium ${ }_{130}$ at $x=x_{S}:=\sqrt{v}$. Stationary distributions of this model are 131 examined in Ref. [35]. For nodes $i=1, \ldots, N$ the network 132 is assumed to evolve according to the stochastic differential 133 equation (SDE),

$$
d x_{i}=\left[f\left(x_{i}, v\right)+\beta \sum_{j \in N_{i}}\left(x_{j}-x_{i}\right)\right] d t+\alpha d w_{i},
$$

${ }_{134}$ where $N_{i}$ are the neighbors that provide inputs to node $i, \beta$ is 135 the coupling strength, $\alpha$ the strength of the additive noise, and ${ }_{136} w_{i}$ are independent Wiener processes.

137

In the case $N=2$ with bidirectional coupling [36], we have

$$
\begin{aligned}
& d x_{1}=\left[f\left(x_{1}, v\right)+\beta\left(x_{2}-x_{1}\right)\right] d t+\alpha d w_{1}, \\
& d x_{2}=\left[f\left(x_{2}, v\right)+\beta\left(x_{1}-x_{2}\right)\right] d t+\alpha d w_{2},
\end{aligned}
$$

${ }_{138}$ where in the noise-free case $\alpha=0$ there are equilibria at $139 x_{Q Q}:=\left(x_{Q}, x_{Q}\right), x_{S S}:=\left(x_{S}, x_{S}\right)$, and $x_{A A}:=\left(x_{A}, x_{A}\right)$ for any $140 \beta$. Up to six more equilibria depend on $0 \leqslant \beta$ and $0<v<1$. 141 The regimes noted in Ref. [36] can be precisely characterized. 142 One can verify that the number of solutions changes at a 143 saddle-node bifurcation when

$$
-27 \beta^{3}+(27 v+9) \beta^{2}-9\left(v+\frac{1}{3}\right)^{2} \beta+v(v-1)=0 .
$$

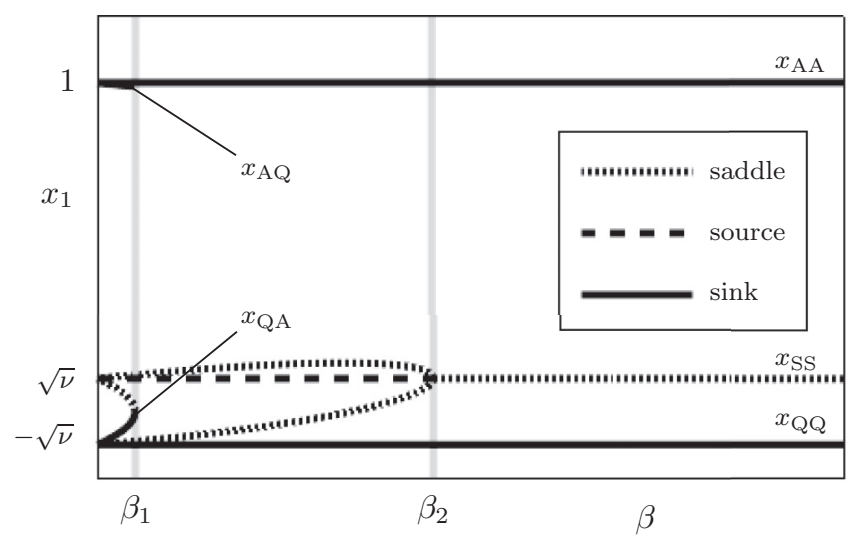

FIG. 1. Bifurcation diagram for the system of two bidirectionally coupled nodes (3) with $\alpha=0$ and $\nu=0.01$ projected into the $\left(\beta, x_{1}\right)$ plane, where $\beta$ is the coupling strength (cf. Fig. 2 in Ref. [36]). We are interested in how the system escapes from the quiescent attracting state $x_{Q Q}$ to the active attracting state $x_{A A}$ under the influence of lowamplitude noise, $0<\alpha \ll 1$. The three regimes that exist in terms of the structures that must be overcome for the transition have parallels in more general cases. In this case they are divided by a saddlenode (fold) bifurcation at $\beta_{1}=0.0101$ and a pitchfork bifurcation of the separating saddles at $\beta_{2}=0.09$. In the weak coupling regime $\beta<\beta_{1}$, the escape will be via an additional attractor, $x_{Q A}$ or $x_{A Q}$, while in the strong coupling ("fast domino" regime) $\beta>\beta_{2}$, the escapes are approximately synchronized and pass near $x_{S S}$. Escapes in the intermediate coupling ("slow domino" regime) $\beta_{1}<\beta<\beta_{2}$ are associated with escape over a symmetry broken saddle.

For small $v$ this implies there is a saddle node for $\beta={ }_{144}$ $\beta_{1}>0$. A pitchfork bifurcation occurs at intermediate $\beta_{2}={ }_{145}$ $\left(\sqrt{v}-4 v+3 v^{3 / 2}\right) /(1-3 \sqrt{v})$. Let $x_{Q S}$ denote the branch of ${ }_{146}$ equilibria that continues from $\left(x_{Q}, x_{S}\right)$ at $\beta=0$. We note ${ }_{147}$ $x_{S A}$ (saddle) and $x_{Q A}$ (stable) meet while simultaneously $x_{A S}{ }_{148}$ (saddle) and $x_{A Q}$ (stable) meet at the saddle node at $\beta_{1}$. The ${ }_{149}$ branches $x_{Q S}$ and $x_{S Q}$ meet $x_{S S}$ at the pitchfork bifurcation 150 at $\beta_{2}$. Observe that there are three qualitatively different ${ }_{151}$ regimes of coupling depending on whether there are nine ${ }_{152}$ $\left(\beta<\beta_{1}\right)$, five $\left(\beta_{1}<\beta<\beta_{2}\right)$, or three $\left(\beta>\beta_{2}\right)$ equilibria. ${ }_{153}$ The bifurcation diagram for $v=0.01$ is shown in Fig. 1: in ${ }_{154}$ this case, $\beta_{1}=0.0101$ and $\beta_{2}=0.09$.

We give an initial condition $x_{i}(0)=x_{Q}$ for (2) and pick a 156 threshold $x_{S}<\xi<x_{A}$. The first escape time of node $i$ is the ${ }_{157}$ random variable $\tau^{(i)}=\inf \left\{t>0: x_{i}(t)>\xi\right\}$ that depends on ${ }_{158}$ the network, the parameters, and the particular noise path: It ${ }_{159}$ has a distribution implied by that of the noise. Independence 160 of the $w_{i}$ means that (with probability one) no two escapes ${ }_{161}$ will occur at the same time and so we can assume there is a ${ }_{162}$ permutation $s(i)$ of $\{1, \ldots, N\}$ such that $\tau^{s(i)}<\tau^{s(j)}$ for any ${ }_{163}$ $i<j$. We denote by $\mathbb{P}(s)$ the probability of a sequence $s$ being ${ }_{164}$ realized and define the time of the $i$ th escape by $\tau^{i}=\tau^{s(i)}$. We ${ }_{165}$ use the convention $\tau^{0}=0$. The time between escapes $j$ and ${ }_{166}$ $k>j$ is denoted $\tau^{k \mid j}=\tau^{k}-\tau^{j}$, with means $T^{(i)}=\mathbb{E}\left[\tau^{(i)}\right]{ }_{167}$ and $T^{k \mid j}=\mathbb{E}\left[\tau^{k \mid j}\right]$. Note that for $\beta=0$ all sequences are ${ }_{168}$ equally likely, meaning $\mathbb{P}(s)=1 / N$ !.

In networks of the form (3), as long as $0<v<1$ so 170 that $x_{Q}$ is linearly stable, the $\tau^{(i)}$ are independent random ${ }_{171}$ variables with exponential tails for $\beta=0$ whose mean can be ${ }_{172}$ 
173 approximated using the one-dimensional Kramers' formula 174 (e.g., Ref. [31]) which states in the limit $\alpha \rightarrow 0$,

$$
T^{(i)} \approx \frac{2 \pi}{\sqrt{V^{\prime \prime}\left(x_{Q}\right)\left|V^{\prime \prime}\left(x_{S}\right)\right|}} e^{\frac{2}{\alpha^{2}}\left[V\left(x_{S}\right)-V\left(x_{Q}\right)\right]} .
$$

175 We show that the distributions $\tau$ and $\mathbb{P}(s)$ change in subtle 176 ways on increasing $\beta$.

177 The persistence of the hyperbolic fixed points and the 178 robustness of connections means there is a weak coupling 179 regime. For small enough $\beta>0$, the quiescent states are 180 perturbed but not destroyed, and the escape of one node 181 modifies the rate of escape of the other nodes. However, the 182 means (4) should vary continuously with the parameter. For 183 the strong coupling (synchronized) regime [26,28], for large ${ }_{184} \beta$, the nodes synchronize and there is a strong dependence, 185 meaning they escape en masse, hence "fast domino." For the 186 intermediate coupling regime, the escape of one node leads 187 to a delayed (but essentially deterministic) response from the 188 other units, hence "slow domino."

${ }_{189}$ We illustrate these differences for (3) in Fig. 2, which 190 shows the behavior of escapes from $x_{Q Q}$ in the weak noise 191 limit with $v=0.01$ fixed and depending on $\beta$, where the 192 SDE is solved using a fixed time-step Heun method. The 193 symmetry in the coupling of the system can be seen as a 194 reflection about the line $x_{1}=x_{2}$. The coupled system (3) can 195 be seen as a noise perturbed potential flow for $\tilde{V}\left(x_{1}, x_{2}\right)=$ $196 V\left(x_{1}\right)+V\left(x_{2}\right)+\frac{1}{2} \beta\left(x_{1}-x_{2}\right)^{2}$ (we suppress the $v$ and $\beta$ 197 dependence). The mean escape time between two minima 198 of the potential can be estimated using a multidimensional 199 Kramers' formula: the mean time from $x^{*}$ to $y^{*}$ over the 200 minimum height pass saddle ("gate") at $z$ is

$$
T\left(x^{*}, z^{*}, y^{*}\right) \approx P\left(x^{*}, z^{*}\right) e^{\frac{2}{\alpha^{2}}\left[\tilde{V}\left(z^{*}\right)-\tilde{V}\left(x^{*}\right)\right]}
$$

201 for $\alpha \rightarrow 0$, where the prefactor $P$ depends on the Hessian $202 \nabla^{2} \tilde{V}\left(z^{*}\right)$ (see, e.g., Ref. [31]). Note that to this leading order $203 T$ is independent of $y^{*}$.

204 We estimate the dependence of mean time $T^{2 \mid 0}=T^{2 \mid 1}+$ ${ }_{205} T^{1 \mid 0}$ of escape for (3) on coupling, where there may be multiple 206 paths of escape. If $\widetilde{T}\left(x^{*}, \tilde{z}^{*}, y^{*}\right)$ is the mean time of escape 207 assuming it takes path $\tilde{z}^{*}$ out of $G$ possible symmetrically 208 equivalent gates, then $\widetilde{T}\left(x^{*}, \tilde{z}^{*}, y^{*}\right)=\frac{1}{G} T\left(x^{*}, z^{*}, y^{*}\right)$, where $z^{*}$ 209 is associated with multiple paths of escape.

210 In the weak coupling regime $0<\beta<\beta_{1}$, each symmetric 211 path is equally probable and so $2 T^{1 \mid 0} \approx \widetilde{T}\left(x_{Q Q}, x_{Q S}, x_{Q A}\right)+$ ${ }_{212} \widetilde{T}\left(x_{Q Q}, x_{S Q}, x_{A Q}\right)$, while $2 T^{2 \mid 1} \approx T\left(x_{Q A}, x_{S A}, x_{A A}\right)+T\left(x_{A Q}\right.$, $\left.213 x_{A S}, x_{A A}\right)$. Hence

$$
T^{2 \mid 0} \approx \frac{1}{2} T\left(x_{Q Q}, x_{Q S}, x_{Q A}\right)+T\left(x_{Q A}, x_{S A}, x_{A A}\right) .
$$

214 In the intermediate coupling regime ("slow domino" 215 regime) $\beta_{1}<\beta<\beta_{2}$, there is a one-step escape process, but 216 there are two possible gates that can be traversed,

$$
T^{2 \mid 0} \approx \frac{1}{2}\left[T\left(x_{Q Q}, x_{S Q}, x_{A A}\right)+T\left(x_{Q Q}, x_{Q S}, x_{A A}\right)\right] .
$$

217 Note that this asymptotic expression will be nonuniform in $\beta$ : 218 near $\beta=\beta_{1}$ there will be a long deterministic delay associated 219 with passage past the region of the saddle node, as is evident 220 in Fig. 2(c).


FIG. 2. Level sets of $\tilde{V}$ (where red corresponds to the most negative) for $N=2$ bidirectionally coupled nodes (3) with fixed $v=0.05$ and four values of $\beta$. The equilibria for $\alpha=0$ are marked as $\bullet$ sinks, $\square$ sources, and $\boldsymbol{\Delta}$ saddles. Typical noise paths starting at $x_{Q Q}$ are shown in each panel computed for (3) and for $\alpha=0.1$. The panels show typical escapes of (a) uncoupled, (b) weakly coupled, (c) intermediate coupled ("slow domino"), and (d) strongly coupled ("fast domino") regimes.

In the strong coupling regime ("fast domino" regime) 221 $\beta>\beta_{2}$, there is a one-step escape process with a unique gate, 222

$$
T^{2 \mid 0} \approx T\left(x_{Q Q}, x_{S S}, x_{A A}\right)
$$

Each of these regimes will give a different scaling in the limit 223 $\alpha \rightarrow 0$, while the scalings at crossovers between regimes are 224 accessible to generalizations of Kramers' formula for passage 225 over nonhyperbolic saddles [31]. This is explored in more 226 detail in Ref. [37], including computing the timing of the 227 escape once the gate has been traversed in the intermediate ${ }_{228}$ and strong coupling regimes.

229

\section{SEQUENTIAL ESCAPES FOR A THREE NODE CHAIN 230}

For a more general network, the sequence of escapes of 231 the network depends not only on the number of nodes that 232 have already escaped but also the sequence in which they 233 escape. We consider a unidirectionally coupled chain of $N=3{ }_{234}$ bistable systems (2) where the input sets $N_{i}$ for node $i$ are given ${ }_{235}$ by $\left(N_{1}, N_{2}, N_{3}\right)=(\{2\},\{3\},\{\})$,

$$
\begin{aligned}
& d x_{1}=\left[f\left(x_{1}, v\right)+\beta\left(x_{2}-x_{1}\right)\right] d t+\alpha d w_{1}, \\
& d x_{2}=\left[f\left(x_{2}, \nu\right)+\beta\left(x_{3}-x_{2}\right)\right] d t+\alpha d w_{2}, \\
& d x_{3}=\left[f\left(x_{3}, \nu\right)\right] d t+\alpha d w_{3} .
\end{aligned}
$$

Figure 3 illustrates the three coupling regimes: the weak cou- 237 pling regime $\left(\beta<\beta_{1}\right)$, intermediate coupling (slow domino) 238 $\left(\beta_{1}<\beta<\beta_{3}\right)$, and strong coupling (fast domino) $\left(\beta>\beta_{3}\right) \quad 239$ 
(a)

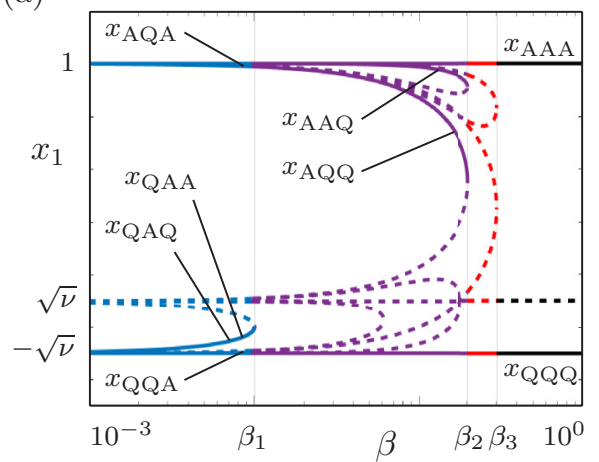

(b)

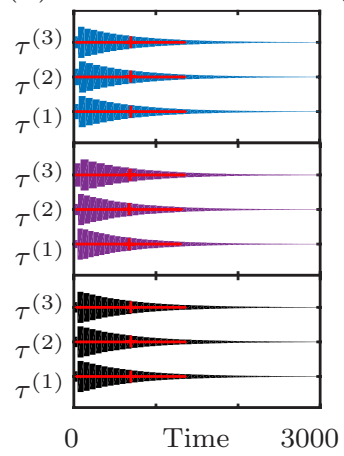

(c)

$(3,2,1)$

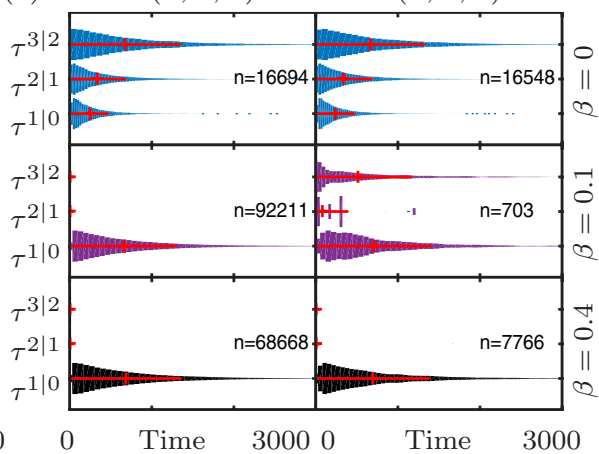

(d)



FIG. 3. (a) Bifurcation diagram showing $x_{1}$ vs $\beta$ (log axis) for (8) with $v=0.01$ and no noise $\alpha=0$ : Dashed branches are unstable. In the weak coupling regime $\left(\beta<\beta_{1}=0.0101\right.$, blue) all branches continue from $\beta=0$. There are two intermediate (slow domino) coupling regimes: For the lower one $\left(\beta_{1}<\beta<\beta_{2} \approx 0.2025\right.$, purple) there are still stable and unstable partially escaped states while for $\left(\beta_{2}<\beta<\beta_{3} \approx 0.3035\right.$, red) there are only partially escaped saddles. For the strong (fast domino) coupling regime $\beta>\beta_{3}$, all equilibria are synchronized in the absence of noise. For (b)-(d) we computed $10^{5}$ samples using $\alpha=0.03$ for $\beta=0$ (blue), 0.1 (purple), and 0.4 (black). (b) shows violin plots of the distribution of escape times $\tau^{(i)}$ of node $i$ : Observe that these change little with coupling. The red cross indicates mean (vertical) and $+/-$ one standard deviation (horizontal). (c) shows the distribution of sequential escape times $\tau^{k \mid k-1}$ for $k=1,2,3$, for sequences $(3,2,1)$ and $(1,2,3)$. The number of samples $n$ (out of $10^{5}$ ) that undergo this sequence of escapes is shown. (d) shows the probability of a given sequence being realized. In the strongly coupled case $\beta=0.4$, the escapes are almost always synchronized, and the most frequent sequence is $(3,2,1)$. The case $\beta=0.1$ and sequence $(1,2,3)$ is an example of a nonsynchronous escape in the intermediate coupling regime; the third escape typically occurs some time after the first two: see Table I.

240 regimes for this system. Note that intermediate coupling can 241 be split further into two subregimes at $\beta_{2}$. There are qualitative 242 changes in the asymptotic behavior of sequential escapes on 243 changing $\beta$, with strongly synchronized escapes for strong 244 coupling.

To characterize the distribution of times of $n$th escape we consider the coefficient of variation of $\tau$ given by

$$
\mathrm{CV}(\tau)=\sigma(\tau) / \mathbb{E}[\tau]
$$

247 where $\sigma(\tau)$ denotes the standard deviation For $\beta=0.0$ (and 248 for all first escapes) we have $\operatorname{CV}\left(\tau^{k \mid k-1}\right) \approx 1$, indicating 249 an exponential distribution. In the intermediate coupling 250 (slow domino) regime $\beta=0.1$, the most likely sequence 251 is $(3,2,1)$ : Considering only this sequence for the data in 252 Fig. 3, we find $\operatorname{CV}\left(\tau^{1 \mid 0}\right)=0.9608, \operatorname{CV}\left(\tau^{2 \mid 1}\right)=0.3308$, and ${ }_{253} \mathrm{CV}\left(\tau^{3 \mid 2}\right)=0.2210$ - after the first (approximately exponen254 tially distributed) escape the remaining escapes are close 255 to deterministic $\left(\mathbb{E}\left[\tau^{2 \mid 1}\right]=4.087, \mathbb{E}\left[\tau^{3 \mid 2}\right]=4.797\right)$. On the 256 other hand, for a rarer sequence $(1,2,3)$ in the intermediate 257 regime, we find $\operatorname{CV}\left(\tau^{1 \mid 0}\right)=0.9783, \operatorname{CV}\left(\tau^{2 \mid 1}\right)=3.662$, and ${ }_{258} \mathrm{CV}\left(\tau^{3 / 2}\right)=1.27$ - after the first exponentially distributed es259 cape there are very large variations in escape time. Finally, 260 in the strongly coupling (fast domino) regime $\beta=0.4$ and 261 the most likely sequence $(3,2,1)$, we have $\mathbb{E}\left[\tau^{2 \mid 1}\right]=0.6568$, ${ }_{262} \mathbb{E}\left[\tau^{3 \mid 2}\right]=0.9664$. Table I gives the probability, mean, and 263 coefficient of variation for sequential escape times of the simulations shown in Fig. 3. Note that as $\beta$ increases, the 264 system remains closer to synchronization, leading to an 265 increasing randomization of the sequence of escapes caused 266 by fluctuations about the synchronized state.

\section{DISCUSSION}

For general heterogeneous networks it is still possible to ${ }_{269}$ classify the interactions between nodes $x_{i}$ and $x_{j}$ as weak, 270 intermediate, or strong depending on whether the escape of 271 node $x_{i}$ modifies the rate of the noise-induced escape of $x_{j}, 272$ whether $x_{j}$ will undergo a deterministic escape in a bounded 273 time, or whether $x_{j}$ will be synchronized in its escape with $x_{i}, \quad 274$ respectively. This will depend on the state of the other nodes 275 that are connected to $x_{i}$ and $x_{j}$, and so the classification of the 276 interaction is, in general, state and sequence dependent. 277

The changes in distribution of timings and sequences of 278 escapes in stochastically perturbed coupled networks can be 279 usefully thought of as an emergent behavior of the network. 280 In particular, even for intermediate or strong coupling where 281 there are no symmetry broken attractors in the noise-free 282 case, the asymptotic behavior of the sequence of escapes 283 is qualitatively different in the low noise limit. A study of 284 such sequential escapes will be of interest in a variety of 285 situations where stochastic forcing of individual sites with 286 asymmetric attractors interacts with the coupling strength to 287 change the sequence of escapes. For example, Ref. [37] uses 288 
TABLE I. Data table. For the simulations shown in Fig. 3, the columns in this table show the sequence of escape, the probability $\mathbb{P}$ that a sequence will be realized, followed by the mean, standard deviation, and coefficient of variation of $\tau^{k \mid k-1}$ conditional on this sequence for $k=1,2,3$.

\begin{tabular}{|c|c|c|c|c|c|c|c|c|c|c|c|c|c|}
\hline Sequence & $\mathbb{P}$ & $\tau$ & $\mathbb{E}(\tau)$ & $\sigma(\tau)$ & $\mathrm{CV}(\tau)$ & $\tau$ & $\mathbb{E}(\tau)$ & $\sigma(\tau)$ & $\mathrm{CV}(\tau)$ & $\tau$ & $\mathbb{E}(\tau)$ & $\sigma(\tau)$ & $\mathrm{CV}(\tau)$ \\
\hline \multicolumn{14}{|c|}{$\beta=\mathbf{0}$ : Uncoupled systems } \\
\hline$(3,2,1)$ & 0.167 & $\tau^{1 \mid 0}$ & 244.53 & 221.98 & 0.91 & $\tau^{2 \mid 1}$ & 334.87 & 340.60 & 1.02 & $\tau^{3 / 2}$ & 673.07 & 668.26 & 0.99 \\
\hline$(3,1,2)$ & 0.166 & $\tau^{1 \mid 0}$ & 245.94 & 222.72 & 0.91 & $\tau^{2 \mid 1}$ & 333.61 & 330.46 & 0.99 & $\tau^{3 / 2}$ & 662.49 & 661.12 & 1.00 \\
\hline$(2,3,1)$ & 0.167 & $\tau^{1 \mid 0}$ & 246.58 & 226.22 & 0.92 & $\tau^{2 \mid 1}$ & 332.64 & 329.08 & 0.99 & $\tau^{3 \mid 2}$ & 668.02 & 674.47 & 1.01 \\
\hline$(2,1,3)$ & 0.167 & $\tau^{1 \mid 0}$ & 243.26 & 223.67 & 0.92 & $\tau^{2 \mid 1}$ & 334.81 & 331.77 & 0.99 & $\tau^{3 / 2}$ & 671.92 & 665.28 & 0.99 \\
\hline$(1,2,3)$ & 0.165 & $\tau^{1 \mid 0}$ & 243.57 & 223.05 & 0.92 & $\tau^{2 \mid 1}$ & 337.94 & 337.15 & 1.00 & $\tau^{3 / 2}$ & 664.35 & 655.76 & 0.99 \\
\hline$(1,3,2)$ & 0.168 & $\tau^{1 \mid 0}$ & 246.26 & 224.39 & 0.91 & $\tau^{2 \mid 1}$ & 329.51 & 329.09 & 1.00 & $\tau^{3 / 2}$ & 667.31 & 667.83 & 1.00 \\
\hline \multicolumn{14}{|c|}{$\beta=\mathbf{0 . 1}$ : Intermediate coupling regime ("slow domino-effect") } \\
\hline$(3,2,1)$ & 0.922 & $\tau^{1 \mid 0}$ & 658.98 & 633.17 & 0.96 & $\tau^{2 \mid 1}$ & 4.09 & 1.36 & 0.33 & $\tau^{3 / 2}$ & 4.80 & 1.06 & 0.22 \\
\hline$(3,1,2)$ & 0.002 & $\tau^{1 \mid 0}$ & 730.13 & 658.49 & 0.90 & $\tau^{2 \mid 1}$ & 2.26 & 1.42 & 0.63 & $\tau^{3 / 2}$ & 1.12 & 1.01 & 0.90 \\
\hline$(2,3,1)$ & 0.024 & $\tau^{1 \mid 0}$ & 652.22 & 611.87 & 0.94 & $\tau^{2 \mid 1}$ & 1.50 & 1.27 & 0.85 & $\tau^{3 / 2}$ & 2.97 & 1.55 & 0.52 \\
\hline$(2,1,3)$ & 0.031 & $\tau^{1 \mid 0}$ & 666.43 & 647.67 & 0.97 & $\tau^{2 \mid 1}$ & 3.54 & 1.70 & 0.48 & $\tau^{3 / 2}$ & 487.84 & 673.65 & 1.38 \\
\hline$(1,2,3)$ & 0.007 & $\tau^{1 \mid 0}$ & 704.30 & 689.06 & 0.98 & $\tau^{2 \mid 1}$ & 82.71 & 302.97 & 3.66 & $\tau^{3 / 2}$ & 509.47 & 647.88 & 1.27 \\
\hline$(1,3,2)$ & 0.014 & $\tau^{1 \mid 0}$ & 703.84 & 663.34 & 0.94 & $\tau^{2 \mid 1}$ & 617.64 & 665.10 & 1.08 & $\tau^{3 / 2}$ & 3.93 & 1.46 & 0.37 \\
\hline \multicolumn{14}{|c|}{$\beta=\mathbf{0 . 4}$ : Strong coupling regime ("fast domino-effect") } \\
\hline$(3,2,1)$ & 0.687 & $\tau^{1 \mid 0}$ & 688.02 & 662.25 & 0.96 & $\tau^{2 \mid 1}$ & 0.66 & 0.38 & 0.58 & $\tau^{3 / 2}$ & 0.97 & 0.40 & 0.41 \\
\hline$(3,1,2)$ & 0.024 & $\tau^{1 \mid 0}$ & 708.41 & 691.41 & 0.98 & $\tau^{2 \mid 1}$ & 0.36 & 0.27 & 0.75 & $\tau^{3 / 2}$ & 0.21 & 0.18 & 0.86 \\
\hline$(2,3,1)$ & 0.128 & $\tau^{1 \mid 0}$ & 690.46 & 682.03 & 0.99 & $\tau^{2 \mid 1}$ & 0.29 & 0.25 & 0.86 & $\tau^{3 / 2}$ & 0.62 & 0.39 & 0.63 \\
\hline$(2,1,3)$ & 0.053 & $\tau^{1 \mid 0}$ & 702.68 & 681.17 & 0.97 & $\tau^{2 \mid 1}$ & 0.41 & 0.31 & 0.76 & $\tau^{3 / 2}$ & 0.50 & 0.53 & 1.06 \\
\hline$(1,2,3)$ & 0.078 & $\tau^{1 \mid 0}$ & 695.96 & 680.09 & 0.98 & $\tau^{2 \mid 1}$ & 4.00 & 49.62 & 12.41 & $\tau^{3 / 2}$ & 0.76 & 0.70 & 0.92 \\
\hline$(1,3,2)$ & 0.030 & $\tau^{1 \mid 0}$ & 694.73 & 651.60 & 0.94 & $\tau^{2 \mid 1}$ & 17.54 & 151.01 & 8.61 & $\tau^{3 / 2}$ & 0.30 & 0.24 & 0.80 \\
\hline
\end{tabular}

289 this to explain some phenomena in the networks of coupled 290 oscillatory bistable units considered in Ref. [22].

291

292 The authors gratefully acknowledge the financial sup293 port of the EPSRC via Grant No. EP/N014391/1. We thank the anonymous referees for their comments, criti- 294 cisms, and suggestions. P.A. gratefully acknowledges fund- 295 ing from the European Union's Horizon 2020 research 296 and innovation programme under the Marie Skłodowska- 297 Curie Grant Agreement No. 643073 for providing opportu- 298 nities to discuss this work with members of the CRITICS 299 network.
[1] P. Jung, U. Behn, E. Pantazelou, and F. Moss, Collective response in globally coupled bistable systems, Phys. Rev. A 46, R1709 (1992).

[2] A. Pikovsky, A. Zaikin, and M. A. de La Casa, System Size Resonance in Coupled Noisy Systems and in the Ising Model, Phys. Rev. Lett. 88, 050601 (2002).

[3] S. Christ, B. Sonnenschein, and L. Schimansky-Geier, Tristable and multiple bistable activity in complex random binary networks of two-state units, Eur. Phys. J. B 90, 14 (2017).

[4] G. Ansmann, K. Lehnertz, and U. Feudel, Self-Induced Switchings Between Multiple Space-Time Patterns on Complex Networks of Excitable Units, Phys. Rev. X 6, 011030 (2016).

[5] J. Emenheiser, A. Chapman, M. Pósfai, J. P. Crutchfield, M. Mesbahi, and R. M. D'Souza, Patterns of patterns of synchronization: Noise induced attractor switching in rings of coupled nonlinear oscillators, Chaos 26, 094816 (2016).

[6] M. Löcher, D. Cigna, and E. R. Hunt, Noise Sustained Propagation of a Signal in Coupled Bistable Electronic Elements, Phys. Rev. Lett. 80, 5212 (1998).
[7] A. A. Zaikin, J. García-Ojalvo, L. Schimansky-Geier, and J. Kurths, Noise Induced Propagation in Monostable Media, Phys. Rev. Lett. 88, 010601 (2001).

[8] J. F. Lindner, S. Chandramouli, A. R. Bulsara, M. Löcher, and W. L. Ditto, Noise Enhanced Propagation, Phys. Rev. Lett. 81, 5048 (1998).

[9] K. Boukheddaden, I. Shteto, B. Hôo, and F. Varret, Dynamical model for spin-crossover solids. II. Static and dynamic effects of light in the mean-field approach, Phys. Rev. B 62, 14806 (2000).

[10] T. Ogawa, Domino mechanisms in photoinduced phase transitions, Phase Trans. 74, 93 (2001).

[11] K. Yonemitsu and K. Nasu, Theory of photoinduced phase transitions in itinerant electron systems, Phys. Rep. 465, 1 (2008).

[12] T. Graf and T. Enver, Forcing cells to change lineages, Nature (London) 462, 587 (2009).

[13] J. Wang, L. Xu, E. Wang, and S. Huang, The potential landscape of genetic circuits imposes the arrow of time in stem cell differentiation, Biophys. J. 99, 29 (2010). 
[14] Y. Chai, F. Chu, R. Kolter, and R. Losick, Bistability and biofilm formation in Bacillus subtilis, Mol. Microbiol. 67, 254 (2008).

[15] M. Chinazzi and G. Fagiolo, Systemic risk, contagion, and financial networks: A survey, SSRN, doi:10.2139/ssrn.2243504 (2013).

[16] P. Gai and S. Kapadia, Contagion in financial networks, Proc. R. Soc. London Ser. A 466, 2401 (2010).

[17] A. G. Haldane and R. M. May, Systemic risk in banking ecosystems, Nature (London) 469, 351 (2011).

[18] M. Summer, Financial contagion and network analysis, Annu. Rev. Financ. Econ. 5, 277 (2013).

[19] P. Ashwin, S. Wieczorek, R. Vitolo, and P. Cox, Tipping points in open systems: Bifurcation, noise-induced and rate-dependent examples in the climate system, Philos. Trans. R. Soc. A 370, 1166 (2012); C. Hobbs, P. Ashwin, S. Wieczorek, R. Vitolo, and P. Cox, ibid. 371, 0098 (2013)

[20] T. M. Lenton, H. Held, E. Kriegler, J. W. Hall, W. Lucht, S. Rahmstorf, and H. J. Schellenhuber, Tipping elements in the earth's climate system, Proc. Natl. Acad. Sci. USA 105, 1786 (2008).

[21] C. A. Boulton, L. C. Allison, and T. M. Lenton, Early warning signals of atlantic meridional overturning circulation collapse in a fully coupled climate model, Nat. Commun. 5, 5752 (2014).

[22] O. Benjamin, T. H. B. Fitzgerald, P. Ashwin, K. TsanevaAtanasova, F. Chowdhury, M. P. Richardson, and J. R. Terry, A phenomenological model of seizure initiation suggests network structure may explain seizure frequency in idiopathic generalised epilepsy, J. Math. Neurosci. 2, 1 (2012).

[23] S. N. Kalitzin, D. N. Velis, and F. H. Lopes da Silva, Stimulationbased anticipation and control of state transitions in the epileptic brain, Epilepsy Behav. 17, 310 (2010).

[24] R. S. Parker and G. Clermont, Systems engineering medicine: engineering the inflammation response to infectious and traumatic challenges, J. R. Soc. Interface 7, 989 (2010).

[25] I. Dobson, B. A. Carreras, V. E. Lynch, and D. E. Newman, Complex systems analysis of series of blackouts: Cascading failure, critical points, and self-organization, Chaos 17, 026103 (2007)

[26] N. Berglund, B. Fernandez, and B. Gentz, Metastability in interacting nonlinear stochastic differential equations: I. From weak coupling to synchronization, Nonlinearity 20, 2551 (2007).

[27] N. Berglund, B. Fernandez, and B. Gentz, Metastability in interacting nonlinear stochastic differential equations: II. Large$N$ behavior, Nonlinearity 20, 2583 (2007).

[28] A. Neiman, Synchronizationlike phenomena in coupled stochastic bistable systems, Phys. Rev. E 49, 3484 (1994).

[29] J. L. Mateos and F. R. Alatriste, Phase synchronization for two Brownian motors with bistable coupling on a ratchet, Chem. Phys. 375, 464 (2010).

[30] Dynamics of Coupled Map Lattices and of Related Spatially Extended Systems, edited by J.-R. Chazottes and B. Fernandez, Lecture Notes in Physics Vol. 671 (Springer, New York, 2005).

[31] N. Berglund, Kramers' law: Validity, derivations and generalisations, Markov Processes Relat. Fields 19, 459 (2013), arXiv:1106.5799.

[32] N. Berglund and B. Gentz, Noise-Induced Phenomena in SlowFast Dynamical Systems, Springer Series on Probability and its Applications (Springer, Berlin, 2006).

[33] H. A. Kramers, Brownian motion in a field of force and the diffusion model of chemical reactions, Physica 7, 284 (1940).

[34] J. M. J. Van Leeuwen, The domino effect, Am. J. Phys. 78, 721 (2010).

[35] H. Malchow, W. Ebeling, R. Feistel, and L. Schimansky-Geier, Stochastic bifurcations in a bistable reaction-diffusion system with Neumann boundary conditions, Ann. Phys. 495, 151 (1983).

[36] M. Frankowicz and E. Gudowska-Nowak, Stochastic simulation of a bistable chemical system: The two-box model, Physica A 116, 331 (1982).

[37] J. L. Creaser, K. Tsaneva-Atansova, and P. Ashwin, Sequential noise-induced escapes for oscillatory network dynamics, SIAM J. Appl. Dyn. Syst. (2017), arXiv:1705.08462. 